\title{
Combined gait disorder: a diagnostic challenge -a case report
}

\section{Ioana Stanescu ${ }^{1}$, Gabriela Dogaru ${ }^{1}$}

1. "Iuliu Hatieganu" University of Medicine and Pharmacy Cluj-Napoca, Romania

2. Clinical Rehabilitation Hospital Cluj-Napoca, Romania

\begin{abstract}
Gait disorders are a major cause of functional impairment and morbidity, especially in the elderly population. Prevalence of gait disorders is higher in persons over 60: is estimated to be around $15 \%$ at 60 years of age and more than $50 \%$ in people $>80$ years. Most gait disorders are multifactorial and have both neurologic and non-neurologic components. Neurological gait abnormalities result from focal or diffuse lesions occurring in the neural pathways linking the cortical motor centers to the peripheral neuromuscular systems. Nonneurological gait abnormalities include gait limitations caused by musculoskeletal, cardiac, or respiratory diseases. Assessment of a gait abnormality should include history, clinical presentation and additional diagnostic tests. Finding the ethiology of a gait disorder could be a challenge for the practitioners in many cases, requiring interdisciplinary cooperation.
\end{abstract}

Key words: gait disorder, neurologic evaluation, hyperuricemia 
Walking relies on a complex, simultaneous interaction of the motor system, sensory control, and cognitive functions. Gait disorders are a major cause of functional impairment and morbidity, especially in the elderly population, where gait impairment is a common complain. Gait troubles increases the risk of falls, fractures, loss of autonomy, institutionalization and even death. Also, gait disturbances in the elderly are a risk factor for future cardiovascular disease and dementia.

Prevalence of gait disorders is higher in persons over 60: is estimated to be around $15 \%$ at 60 years of age and more than $50 \%$ in people $>80$ years old [1]

Classification of gait abnormalities could be based on clinical features of gait (associated signs and symptoms) or on etiology of gait disorder (underlying cause) [2]

Risk factors for gait and balance disturbances include old age, vascular risk factors (e.g. high blood pressure or diabetes).

Occurrence of cerebrovascular disease in the elderly, particularly of cerebral small vessel disease, also appears to play some role in the development of gait and balance impairment.

Most gait disorders are multifactorial and have both neurologic and non-neurologic components. Neurological gait abnormalities result from focal or diffuse lesions occurring in the neural pathways linking the cortical motor centers to the peripheral neuromuscular systems.

Non neurological gait abnormalities include gait limitations caused by musculoskeletal, cardiac, or respiratory diseases (eg arthritis, cardiac disease, chronic lung disease, and peripheral vascular disease).

In The Einstein Aging study which includes persons aged 70 and older, $35 \%$ of study participants were diagnosed with abnormal gaits: $41,6 \%$ presented with neurological, 48,2\% with non-neurological and $10,1 \%$ with combined gait abnormalities [3]. In this population the prevalence of nonneurological gait abnormalities was higher than the prevalence of neurological gait abnormalities, including combined (20.8\% versus $15.7 \%$ ). Causes of pure nonneurological gait abnormalities included in more than $80 \%$ of cases arthritis or joint deformities, and less frequent chronic lung disease, angina pectoris, cardiac failure and peripheral vascular disease. Causes of neurological gait troubles include ataxic gait - the most frequent, followed by hemiparetic , frontal, parkinsonian and neuropathic gait. [3].

In the population-based Bruneck Study cohort (2), which includes persons of 60 to 97 years, gait abnormalities have almost the same prevalence (32.2\% of study population), but neurological gait disorders were more common (24.0\% versus $17.4 \%$ non-neurological gait abnormalities) in this study population. In this study, nonneurological gait disorders were more frequent in females and occurred predominantly due to orthopaedic reasons.

Common causes of disturbed gait in the elderly are neurological deficits, including sensory deficits (e.g. peripheral neuropathy and vestibulopathy), neurodegeneration (e.g. cerebellar ataxia and parkinsonian syndromes), cognitive impairment (e.g. degenerative dementia), joint degeneration (e.g. coxarthrosis) and general loss of muscle mass (sarcopenia). Furthermore, a fear of falling also contributes to the gait disorder [4] .

Assessment of a gait abnormality should include history, clinical presentation and additional diagnostic tests [5].

History should mention duration and course of gait disorder (episodic or continuous, sudden onset or slow progression), precipitating and exacerbating factors (darkness, fobia), accompanying symptoms and signs (dizziness, pain, etc), associated diseases (diabetes, heart failure), medication use and frequency of falls [6].

Clinical presentation allows recognition of a typical gait pattern, such as hemiparetic / spastic, ataxic, steppage, parkinsonian, dyskinetic, frontal, anxious or psychogenic gait [5]. This includes precise 
clinical tests, such as "timed up-and-go test", pull-test, measure of step length, walking speed, etc.

Additional paraclinical investigations most frequently used are neuroimaging studies, measure of nerve conduction velocities, vestibular and visual testing. New diagnostic modalities have evolved: dual tasking (patient is asked to walk while performing either a cognitive or a motor task), video gait analysis and functional neuroimaging (fMRI and ${ }^{18}$ FDG-PET).

After using this complex algorithm in the management of a gait disorder, finding the precise cause and the appropriate treatment could be a challenge for the practitioners, and implies a multidisciplinary approach.

We present the case of a 81-year old retired teacher, which complains of a sudden gait disorder with onset in new year eve. Gait became impossible, the patient fell severe unsteadiness, he can walk only with the aid of 2 persons, and drag his right leg. A fall is reported with a right ankle strain and after this episode, the patient refuses to walk.

His medical history include diabetes mellitus, arterial hypertension, hyperuricemia and Alzhemier dementia. The patient is taking metformine, aspirin, allopurinol, candesartan and rivastigmine for his medical conditions.

A previous CT scan was performed 2 years ago for a minor cranio-cerebral tramatism after an accidental fall, finding important cortical atrophy and normal pressure hydrocephalus, but the neurosurgical opinion at that time was for conservative treatment, and did not provide any indication for shunting.

The patient refuses to go at the hospital, and remain at home for the first 24 hours, being monitored by the family. Blood pressure was $130 / 80 \mathrm{mmHg}$, glycemia was $145 \mathrm{mg} / \mathrm{dl}$, no motor deficit was noted on the bed plane, the patient can sit without support, eats normally, has no sphincterian incontinence, was conscious, but has confusional episodes in the evening, as he usually has. He sleeps well at night. Swelling of his right ankle was noted, and the patient complains of pain in both ankles and in the left knee.

Because the gait trouble did not improve, on next day the patient was admitted to the emergency department of the local hospital with stroke suspicion.

The clinical examination reveals at admission $\mathrm{BP}=140 / 80 \mathrm{mHg}$, rhythmic heart rate $80 / \mathrm{min}$, peripheral pulses were present al lower limbs, skin pallor, painful tumefaction at the right ankle, without ecchimosis, .

The neurologic examination reveals a conscient and oriented patient, cranial nerves examination was normal, in the upper limbs no motor deficit was detected, a mild motor deficit of $4 / 5$ BMRC grade is noted at right lower limb, but the osteotendinous reflexes were normal and symmetrical and the plantar responses were in flexion. No coordination troubles and no sensory disturbancies were noted. The patient can stand only with the aid of 2 persons and cannot walk at all.

Biochemical examinations confirm hyperglycemia at $149 \mathrm{mg} / \mathrm{dl}$, elevated values of $\mathrm{CK}$ at $608 \mathrm{UI} / \mathrm{L}$, normal values of cholesterol and triglycerides, normal ionogram and elevated values of uric acid at $8,28 \mathrm{mg} / \mathrm{dl}$. Hemogram were normal, and C reactive Protein (CRP) was mildly elevated $(1,5 \mathrm{mg} / \mathrm{dl})$.

Emergency cerebral CT scan found no acute ischemic lesions, cortical atrophy with related dilatation of the ventricular system, diffuse ischemia of white matter (leukoaraiozis). Cerebral MRI confirms the small vessel disease with lacunes and diffuse ischemia of the white matter, cortical atrophy with "ex vacquo" hydrocephalus, but no acute ischemic lesions ware detected of diffusion / perfusion sequences.

Radiography of the right ankle reveals signs of an old fracture of the internal malleola.

The psychologic examination confirms moderate cognitive dysfunction, loss of autonomy (GAFS score of 20, MMSE score of 18/30), an important memory deficit 
(level 1 of 5), but with normal executive functions (BREF score of 16/18).

The patient receives aspirin, fraxiparine, metformin, rosuvastatin and rivastigmine.

Three days later, the gait and stance disorder remains unchanged, but right ankle joint and left knee joint became swollen, eritematous and very painfull. CRP was elevated at $3.3 \mathrm{mg} / \mathrm{dl}$ and uric acid at 10,34 $\mathrm{mg} / \mathrm{dl}$. An acute gout attack was suspected and treated with colchicine, allopurinol and high doses of. NSAIDs.

After treatment and pain resolution, kinesitherapy was initiated. After 5 days, the patient improves his neurologic condition: he could stand with unilateral support, and begin to walk with unilateral assistance, and no motor deficit in his lower limbs was noted.

The patient and his family has give their consent for publishing his clinical data.

Although this patient had major neurologic conditions which could lead independently to a gait disorder of neurologic cause (cerebral small vessel disease, Alzheimer dementia, hydrocephalus), the actual cause of his gait disorder was a non neurologic condition. Evaluation of the etiology of a gait disorder should include besides a complete neurological examination, an osteo-articulary and metabolic evaluation.

\section{BIBLIOGRAPHY}

1. Pinter D, Ritchie SJ, Doubal F, Gattringer T, Morris Z, Bastin ME, del C. Valdés Hernández M, Royle NA, Corley J, Muñoz Maniega S, Pattie A, Dickie DA, Staals J, Gow AJ, Starr MJ, Deary IJ, Enzinger C, Fazekas F, Wardlaw J, Impact of small vessel disease in the brain on gait and balance. Sci Rep. 2017; 7: 41637

2. Mahlknecht P. et al. Prevalence and Burden of Gait Disorders in Elderly Men and Women Aged 60-97 Years: A PopulationBased Study. PLoS One 8, 1-7 (2013).

3. Verghese J, LeValley A, Hall CB, Katz MJ, Ambrose AF, Lipton RB. Epidemiology of gait disorders in community-residing older adults. J Am Geriatr Soc. 2006 Feb; 54(2):255-61

4. Jahn K, Heinze C, Selge C, Heßelbarth K, Schniepp R. Gait disorders in geriatric patients. Classification and therapy. Nervenarzt. 2015 Apr; 86(4):431-9

5. Jahn K, Zwergal A, Schniepp R. Gait disturbances in old age: classification, diagnosis, and treatment from a neurological perspective. Dtsch Arztebl Int. 2010 Apr;107(17):306-15

6. Snijders AH, van de Warrenburg BP, Giladi N, Bloem BR. Neurological gait disorders in elderly people: clinical approach and classification. Lancet Neurol. 2007 Jan; $6(1): 63-74$ 